\title{
RETOS DEL PROCESO DE INTEGRACIÓN EUROPEO. UNA VALORACIÓN
}

Por: José Manuel Saiz Álvarez*

\begin{abstract}
Resumen
Las recientes ampliaciones comunitarias realizadas hacia el Este del continente europeo están provocando una serie de cambios estructurales que serán irreversibles en el tiempo. Para que el proceso de cambio sea beneficioso, desde una doble perspectiva económica y social, se ha de dar dentro de códigos de buen gobierno por parte de las empresas, lucha contra el fraude y la corrupción y la generalización de una economía social de mercado en el que los fallos de mercado sean compensados con un ligero intervencionismo estatal. Experiencia europea que puede servir de ejemplo para otros procesos de integración que se están dando en otras regiones del planeta.
\end{abstract}

\section{Palabras clave}

Unión Europea, fraude, buen gobierno, economía social de mercado, libre comercio

\section{Europa y la Tercera Revolución Postindustrial}

E Curopa está viviendo momentos de cambio. Un proceso de transformación que será irreversible en el tiempo y cuyos resultados los estamos viendo hoy. La creación de una moneda única europea, ya adoptada por trece países europeos; las dos ampliaciones, la primera en 2004 y la segunda en 2007, hacia los Países de Europa Central y Oriental (PECO) ${ }^{1}$, así como la generación de riqueza y bienestar económico y social para todos, está generalizando la llamada Tercera Revolución Postindustrial ${ }^{2}$, posible gracias a la generalización de las Tecnologías

\footnotetext{
* Coordinador del Módulo Europeo «Jean Monnet» de la Universidad Antonio de Nebrija.

1 En la primera, cuya entrada en vigor fue el 1 de mayo de 2004, se incorporaron a la UE ocho países PECO (Eslovaquia, Eslovenia, Estonia, Hungría, Letonia, Lituania, República Checa y Polonia) y dos países mediterráneos (países MED)(Chipre y Malta). El 1 de enero de 2007 entraron los llamados Balcanes-2 (Bulgaria y Rumania).

2 La primera se inició en el siglo XVIII en el Reino Unido con la revolución industrial. La segunda empezó a comienzos del siglo XX con la generalización de los «mass media».
} 
de la Información y las Comunicaciones (TIC), puesta en marcha a inicios de la década de 1990. Dicha Revolución ha llevado, a su vez, hacia una mayor intercomunicación en tiempo real a escala planetaria medible, entre otros factores, por la ley de Moore ${ }^{3}$. Únicamente, gran parte del continente africano, queda fuera de este proceso de globalización económica basado en el conocimiento y en los procesos de $1+D+i^{4}$.

Estos momentos de cambio de la UE, también se reflejan en el mercado de trabajo, al apoyarse en todos los países en la existencia de microempresas y PyME, tanto más cuanto menor sea el grado de desarrollo del mismo. Así, las empresas que forman parte de la llamada economía social, conformada por lo que se conoce como el «Tercer sector», está cobrando una tímida importancia en todo el mundo; las organizaciones que forman parte de dicha economía se caracterizan por ser intensivas en factor trabajo, pero no tanto, en el acceso a TIC avanzadas.

La existencia de economía social es especialmente palpable en el Tercer y el Cuarto mundos, sobre todo por los altos niveles de pobreza existentes en el continente africano, en donde la economía social basada en la solidaridad y en los valores éticos (defensa del más pobre, búsqueda del bienestar individual y social) y religiosos (cumplimiento de una vocación y santificación por medio del amor y de la entrega por medio de Cristo a los más pobres y desheredados de la tierra) ha sustituido, de forma parcial e, incluso total, en algunos países subdesarrollados, al modelo neoliberal.

\footnotetext{
3 Expresa que el número de transistores en un ordenador se duplica, aproximadamente, cada dos años, lo que duplica la velocidad y disminuye el coste.

4 Investigación, desarrollo e innovación. La inclusión de la innovación viene dada por la llamada Estrategia de Lisboa que tiene como objetivo convertir a la UE en la región más dinámica y competitiva del mundo para el año 2010 .
}

\section{A bstract}

The most recent expansions to the Eastern part of the EU are provoking a series of structural changes that are irreversible in time. For the changing process to be benefitial, from an economic and social perspective, is supposed to be in the limits of the given codes of good government by companies, the fight against fraud and corruption and the generalization of a social market economy in which all market failures are compensated with a slight official intervention. This European experience could be an example for other processes of integration that are taking place around world regions.

\section{KEY WORDS}

European Union- fraud- good government- social market economy-free trade. 
Economía social que determina tanto la organización social de los países, como su grado de crecimiento y desarrollo. Sin embargo, y, a pesar de los intentos para evitarlo, el continente africano no logra salir de su extrema pobreza por la existencia de continuas guerras civiles, las escasas oportunidades de inversión, la formación de unos mercados de capitales ineficientes, el escaso acceso a los mercados mundiales y el fenómeno, cada vez más extendido, de la corrupción generalizada.
Conscientes de este hecho, la UE está implementando planes preferenciales de desarrollo con África, tanto con los países del Magreb (Marruecos, Túnez, Libia, Argelia, Mauritania y la República Árabe Saharaui Democrática ${ }^{5}$ ) quienes gozan de regímenes comerciales preferenciales con la UE para productos agrícolas y textiles, principalmente, (Acuerdo de Agadir, 2001) como con América Latina.

\section{LAS RELACIONES UE-AmÉRICA LATINA: UN OBJETIVO POR EXPANDIR}

\section{I}

as relaciones exteriores de la UE con América Latina se caracterizan por la firma de Acuerdos Marco por bloques regionales, tanto con el Tratado de Libre Comercio de América del Norte (TLCAN) a través de México (Tratado de Libre Comercio UE-México (TLCUEM)), y el Mercado Común del Sur (MERCOSUR) a través de Argentina, Brasil y Uruguay (Acuerdo UEMercosur), como con el Mercado Común Centroamericano y la Comunidad Andina. Aeste respecto, y en cuanto a la Comunidad Andina, el 23 de abril de 1993 la UE firmó en Copenhague (Dinamarca) un Acuerdo Marco de Cooperación entre la entonces Comunidad Económica Europea (CEE) y los firmantes del Acuerdo de Cartagena (Bolivia, Ecuador, Perú y Venezuela). Este acuerdo, llamado de tercera generación, amplió el margen de maniobra con respecto al primer acuerdo no preferencial firmado en 1983. El nuevo Acuerdo Marco se ratificó en Colombia mediante la Ley 183 de 23 de enero de 1995 y en la UE mediante la Decisión 98/278 del Consejo de 7 de abril de 1998.
De forma complementaria a este Acuerdo Marco, el 15 de diciembre de 2003 se firmó en Roma (Italia) un Acuerdo de Diálogo Político y Cooperación entre la UE y la Comunidad Andina para fortalecer, mediante la cooperación y el diálogo político, las relaciones políticas y económicas entre ambas orillas del Atlántico. Dicho Acuerdo reemplazó al Acuerdo Marco de Cooperación de 1993, el cual se vería ampliado en 1996 con un Acuerdo entre la UE, la Comunidad Andina y México para combatir la manufactura ilícita de drogas y sustancias psicotrópicas, así como para ejecutar ayuda financiera y técnica a Colombia (Acuerdo de 14 de diciembre de 2000) e incluirla, además, dentro del Sistema de Preferencias Generalizadas (SPG Plus)(Reglamento 980/ 2005 del Consejo) para el período 2006-2009.

Uno de los objetivos de la UE es la lucha contra la pobreza generalizada en el Tercer y el Cuarto Mundos. Sin embargo, la llamada economía social tiene una presencia testimonial en la

\footnotetext{
5 En parte ocupado desde 1975 por Marruecos desde la llamada Marcha Verde, está reconocido por 48 Estados de los que trece tienen una embajada. Está pendiente desde su ocupación la celebración de un referéndum de autodeterminación a partir de una consulta popular (Plan Baker).
} 
UE. Así, por ejemplo, en España, los casi 2,5 millones de personas que trabajan en economía social sólo aportaron en el año 2006 el 7\% del PIB. Por esta razón, la economía neoliberal, basada en la libre competencia entre microempresas, PyME y grandes corporaciones, es la que determina la senda de evolución en términos de crecimiento económico y de bienestar social en los países desarrollados, unido a una fuerte intervención estatal reductora de desequilibrios económicos y sociales. De ahí, que en la UE se haya empezado a hablar de economía social de mercado, más que de una mera economía de mercado.

\section{LA LUCHA CONTRA LA CORRUPCIÓN EN LA UE}

$\mathrm{L}_{\mathrm{a}}$ a creciente competencia entre las corporaciones y el deseo de sus «stakeholders» (clientes, proveedores, accionistas, personal, Consejo de Administración,...) de aumentar de forma continuada en el tiempo el valor de la empresa, puede generar la adopción de prácticas ilícitas y desleales y, por ende, distorsionar, las leyes de la libre competencia. Estos problemas se vuelven estructurales cuando confluyen intereses económicos y políticos en la consecución de objetivos en regímenes dictatoriales, con independencia de su signo político.

Las empresas que desean aumentar su volumen de facturación de forma ilegal, adoptan las siguientes políticas:

- «Dumping» económico. Utilizado tras la Segunda Guerra Mundial, viene definido por la puesta en marcha de subvenciones a fondo perdido por parte de los poderes públicos hacia sus empresas para mejorar así la balanza comercial mediante un aumento del flujo de exportaciones. Este tipo de práctica, utilizada de forma generalizada en Asia, está prohibida por la Organización Mundial de Comercio (OMC), especialmente a partir de la llamada Declaración Ministerial de Doha, cuyas deliberaciones acerca de los principios fundamentales de la OMC dentro del Grupo de Trabajo sobre la interacción entre comercio y política de competencia se han centrado en la no discriminación, la transparencia y el debido proceso. Las prohibiciones de medidas gubernamentales relativas a exportaciones e importaciones ya vienen reflejadas en el artículo XI del GATT («General Agreement on Trade and Tariffs») ${ }^{6}$, como antecedente del Acuerdo General sobre Comercio y Servicios, así como en el artículo IX del GATS ( General Agreement on Trade and Services»), como antecedente de la $\mathrm{OMC}$. En estos se establecen las bases para las consultas sobre prácticas privadas anticompetitivas que contradicen las obligaciones contraídas en el acuerdo.

- "Dumping» social. Generalizado durante la década de 1980. Se establece en aquellos países en donde es mínimo el grado de protección hacia los trabajadores, ya sea por la ausencia de mecanismos de protección social (seguridad Social, sindicatos, legislación laboral protectora,...) o por la existencia de sueldos muy reducidos; salarios que ni

\footnotetext{
${ }^{6}$ La introducción del artículo XI del GATT «en el borrador de Ginebra de 1947 representó un hito trascendental en la diplomacia económica internacional [...] Con el paso de los años, gran parte de la diplomacia del GATT en el campo de la política comercial ha estado relacionada con la aplicación (o no aplicación) gradual del Artículo XI, desde los debates sobre las limitaciones voluntarias de la exportación, las excepciones sectoriales en la agricultura y los textiles,...» (Mathis, 2003: 55).
} 
siquiera llegan a cubrir el mínimo vital en países económicamente más desarro-llados. La Organización Internacional del Trabajo (OIT) prohibió dicho «dumping», por «provocar desempleo y congelación salarial en aquellos sectores económicos de los países desarrollados en los que existe una utilización intensiva de mano de obra (textil, calzado, gama intermedia de informática,...)» (García Menéndez, 1996: 139).

- "Dumping» ecológico. Puesto en marcha durante la década de 1990 tras el endurecimiento de las normas sobre protección del medio ambiente ${ }^{7}$ aprobadas en los países más desarrollados del mundo, incluida la Unión Europea (UE). Como resultado, se instalan plantas industriales (de producción o de ensamblaje) en el Tercer y el Cuarto Mundos, para la creación de empleo y riqueza local y regional, a pesar del impacto negativo sobre el medio ambiente.

- "Dumping» carcelario. Introducido en la R.P. China a finales del siglo XX; , consiste en la utilización de presidiarios como mano de obra muy barata, así como su sobreexplotación para la rendición de penas. Es muy desconocido el impacto económico de dicho tipo de dumping por la ausencia de información que existe sobre el mismo.

- Cartel. Consiste en un acuerdo formal entre varias empresas, grupos de empresas o Gobiernos, que tiene como objeto llegar a acuerdos en precios y cantidades que se deben producir y/o distribuir, para así maximizar sus beneficios y eliminar la competencia en un determinado mercado. Este tipo de prácticas perjudica a los consumidores últimos del producto, al evitar, en muchos casos, la libre competencia.

- Abuso de posición dominante. Viene determinado por el poder de mercado ejercido, por parte de empresas líderes en cada uno de los sectores, tanto en países desarrollados como en vías de desarrollo. Dicha práctica reduce la creación de nuevas empresas e impide la formación de pliopolios ${ }^{8}$ entre las empresas.

De forma complementaria a estas prácticas ilícitas desde un punto de vista económico, también se realizan prácticas de comercio paralelo que consisten en una forma de arbitraje comercial de un producto sujeto a la protección de derechos de propiedad intelectual, desde un mercado donde se ha puesto en circulación originariamente, a otro mercado de destino, haciendo uso de una cadena de distribución paralela a la originaria. Dicha práctica (cuando se realiza el proceso de distribución comercial a varios países) está prohibida en la UE, por discriminar a los consumidores en función de su poder adquisitivo y por provocar, en muchas ocasiones, problemas de desabastecimiento en los mercados con menor poder adquisitivo. Con el objetivo último de evitar estas prácticas ilícitas, en la UE se lucha contra el delito tanto mediante Europol y la OLAF (Office Européen de Lutte Anti-Fraude) $)^{9}$, creada por la Decisión de la Comisión de 28 de abril de 1999 (DOCE L 136/20 de 31 de mayo de 1999), como mediante

\footnotetext{
7 Al ser muy elevada la conciencia medioambiental en Europa, son múltiples las políticas de protección medioambiental existentes en la Unión Europea (UE), entre las que se destacan los programas LIFE (en su triple vertiente: LIFENaturaleza, LIFE-Medio Ambiente y LIFE-Terceros países), Energía inteligente, programa SAVE, ALTENER, COOPENER, STEER,... Programas cuya aplicación y seguimiento viene efectuada a través de la Agencia Europea del Medio Ambiente en funcionamiento desde 1990.

8 Se dividen en pliopolios positivos (creación de empresas, ya sea como resultado de incubadoras de empresas como por división de las mismas para formar otras nuevas) y pliopolios negativos (fusión y adquisiciones de empresas). Sobre este particular, véase Saiz (2004b)

$9 \quad$ Véase Saiz (2006) para una mayor profundización en este tema.
} 
legislación específica sobre la protección de intereses financieros de la UE (art. 274 (exArt. 205) y art. 280 (ex Art. 209a) del Tratado de la UE y Regulación del Consejo 2988/95, de 18 de diciembre, sobre la protección de los intereses financieros de las Comunidades Europeas (Regulación PIF)(DOCE L 312 de 23 de diciembre de 1995) ${ }^{10}$.

Esta defensa jurídica frente a las prácticas ilícitas en la UE, desde una perspectiva de política económica, se caracteriza por la separación entre los intereses económicos y las motivaciones políticas en todos los niveles, lo que es, además, una de las características adicionales del proceso de unificación europeo, sobre todo tras la firma del Tratado de Maastricht en 1992. Al seguir el modelo alemán (modelo Buba) ${ }^{11}$ en lo económico, el cual se caracteriza por su transparencia informativa y por la separación entre los objetivos políticos y económicos para evitar así prácticas de corrupción política y económica, la Unión Europea (UE) se ha convertido en un paradigma de imitación para otros procesos de integración en el planeta.

\section{LA IMPORTANCIA DE LOS BIENES INTANGIBLES EN LA UE}

\begin{abstract}
$\mathcal{J}$ unto a esta lucha contra la corrupción, en el mundo empresarial está cobrando una mayor importancia la existencia de bienes intangibles (formado, entre otros, por la marca, reputación corporativa, capital intelectual, cultura organizacional y patentes) y la fidelización de directivos, profesionales y empleados que trabajan en las corporaciones. Hoy en día, los activos intangibles
\end{abstract}

se han convertido en fuentes principales del valor de las empresas, incluyendo incluso la reputación a la supervivencia de la misma (Gray y Balmer, 1998).

Los recursos intangibles se dividen en humanos (capital intelectual) y no humanos (reputación, marca, patentes, cultura,...) de tal forma que la

10 A esta legislación se unen las (1) Reglas de procedimiento para los casos de trabajo, regulados tanto por la Regulación (Comunidad Europea) 1073/1999 del Parlamento y Consejo Europeos sobre las actividades de la OLAF (DOCE L 136/1 de 31 de mayo de 1999), como por la Regulación (EURATOM) 1074/99, del Parlamento y Consejos Europeos de 25 de mayo, sobre las actividades de la OLAF (DOCE L 136/8 de 31 de mayo de 1999); (2) Las inspecciones horizontales (Regulación del Consejo (EURATOM, CE) 2185/96, de 11 de noviembre (DOCE L 292 de 15 de noviembre de 1996) y la Regulación del Consejo (CEE) 595/91, de 4 de marzo (DOCE L 67 de 14 de marzo de 1991); (3) El control de las ayudas otorgadas tanto para la Política Agrícola Común (PAC) mediante la Regulación del Consejo (CE) 1290/2005, de 21 de junio (DOCE L 209, de 11 de agosto de 2005 corregida en DOCE L 58, de 28 de febrero de 2006), el Reglamento (CEE) 595/91 del Consejo, de 4 de marzo (DOCE L 67, de 14 de marzo de 1991) y la Regulación del Consejo (CE) 1258/1999, de 17 de mayo (DOCE L 160/ 103, de 26 de junio de 1999); como para los Fondos Estructurales, por la Regulación del Consejo 1083/2006, de 11 de julio (DOCE L 210, de 31 de julio de 2006); (4) La recuperación de las ayudas mal concedidas por la UE que se realiza a través de la Regulación de la Comisión 1848/2006 de 14 de diciembre (DOCE L 355 de 15 de diciembre de 2006), la Regulación de la Comisión (CE) 1828/2006 de 8 de diciembre (DOCE L 371/1, de 27 de diciembre de 2006), la Regulación del Consejo (CE, EURATOM) 2028/2004, de 16 de noviembre (DOCE L 105, de 13 de abril de 2006), Regulación del Consejo (CE, EURATOM)1150/2000, de 22 de mayo (DOCE L 130/1, de 31 de mayo); (5) Investigaciones de fraudes internos de la UE que se realiza mediante el Acuerdo Interinstitucional de 25 de mayo de 1999 entre el Parlamento Europeo, el Consejo de la UE y la Comisión de las Comunidades Europeas (DOCE L 136/15, de 31 de mayo de 1999); y (6) Los instrumentos convencionales (Tercer pilar) mediante las Convenciones europeas de 26 de julio de 1995 (Convención PIF)(DOCE C 316, de 27 de noviembre de 1995) y de 26 de mayo de 1997 (DOCE C 195, de 25 de junio de 1997).

11 Es la denominación popular del Banco Central alemán («Deutsche Bundesbank»). Al basarse en este modelo, en el área euro está prohibida la realización de políticas económicas a dos manos («Two-hand Economic Policy») por lo que se separa la política monetaria de la fiscal. Esta prohibición ya quedó establecida en 1992 en el Tratado de Maastricht o Tratado de la Unión Europea. 
empresa puede entenderse como aquel conjunto de interacciones entre activos tangibles e intangibles con el fin de lograr sus objetivos, no necesariamente monetarios, en el caso, por ejemplo, de las Organizaciones sin Ánimo de Lucro (ONG). Uno de los aspectos más importantes de los activos intangibles es que descansan en la información y en las expectativas. Esto último es especialmente observable en los valores que cotizan en Bolsa y, sobre todo, en los denominados 'chicharros' («small caps»). Por ello, los derechos de propiedad de estos recursos no siempre están bien definidos (Navas y Guerras, 1998) y son muy volátiles en el tiempo.

Por otra parte, en la valoración de los activos intangibles está teniendo una creciente importancia la reputación corporativa, como complemento a una mera Responsabilidad Social de la Empresa (RSE), sobre todo, dado el creciente proceso de globalización económica en el que está inmerso el mundo desarrollado. La existencia de mercados, (potenciales o efectivos), más grandes conlleva un mayor riesgo, así como un creciente coste de oportunidad por la posible pérdida de negocios o de posibilidades para mejorar la posición estratégica de la empresa dentro de su sector o industria. En esta situación, no sólo es necesaria la figura del emprendedor con una adecuada visión de futuro; también, los poderes públicos han de apoyar a la empresa con políticas de ayudas no reembolsables, al crear riqueza en las corporaciones de su entorno más inmediato.

Existen dos aproximaciones teóricas sobre la reputación corporativa, ya sea (1) en función de la capacidad relacional de la empresa para satisfacer las expectativas de sus stakeholders
(Fombrun y Shanley, 1990), como (2) según sea el estado de consolidación definitiva de la imagen corporativa de la empresa «tanto en los aspectos de comportamiento como en los informativos» (Quevedo, 2003). Una de las claves para cimentar una buena reputación corporativa que sea sostenible en el tiempo consiste en la puesta en marcha de buenas prácticas caracterizadas por un comportamiento ético, no sólo por la estrategia corporativa en sí, sino también por los profesionales que trabajan en la empresa. Hoy en día, con independencia del sector que se considere, las empresas compiten entre sí para obtener los mejores profesionales, al ser, en muchas ocasiones, incluso cuestión de propia supervivencia.

La globalización económica actual está favoreciendo el agrandamiento de una brecha entre el Tercer y el Cuarto mundos y los países que forman el $\mathrm{G}^{12}$. En la formación de estas diferencias tiene una creciente importancia la puesta en marcha de políticas de dumping, en su múltiple vertiente económica, social, ecológica y carcelaria. Aunque dicha práctica empresarial está prohibida tanto por la Organización Mundial de Comercio (OMC), por vulnerar las leyes de la libre competencia, así como por la Organización Internacional del Trabajo (OIT), por llevar a cabo prácticas de explotación laboral, incluida la explotación infantil, es realizada en el Tercer Mundo, principalmente en Asia, con el beneplácito de las autoridades locales quienes ven en el capital extranjero una vía para escapar de la extrema pobreza.

La mano de obra (legalmente desprotegida) cobra gran importancia (además de las TIC) en la puesta marcha de prácticas ilicitas. En este caso la tecnología no juega un papel

\footnotetext{
${ }^{12}$ Formado por los jefes de Estado y de Gobierno de Canadá, Francia, Alemania, Italia, Japón, Rusia, Reino Unido y Estados Unidos, tiene como objetivo constituir un foro informal de debate y de intercambio de opinión sobre asuntos económicos, sociales y políticos de actualidad. Sin embargo, al representar más del 65 por ciento del PIB mundial, constituye, en realidad, un «lobby» en donde se dirime buena parte de la política y economía mundiales. Desde la primera reunión en la localidad francesa de Rambouillet, y presidida por Valéry Giscard d'Estaing, del 15 al 17 de noviembre de 1975, las reuniones de alto nivel del G8 se han realizado anualmente. A título de curiosidad, ya se saben cuáles van a ser las ciudades que van a albergar las reuniones anuales del G8 hasta el año 2013.
} 
fundamental, al ser los procesos más intensivos en mano de obra y, muy raramente, en factor capital (entendido éste último como capital económico y no capital humano). Se da así una fuerte correlación entre la ausencia de libertades políticas y económicas, situación generalizada en el Tercer y el Cuarto mundos, al no darse las cuatro características propias de una democracia: «(1) la definición de las libertades públicas y de garantías privadas inherentes a los Derechos Humanos; (2) la existencia de un parlamento legislativo elegido por sufragio universal; (3) la separación de poderes entre el
Parlamento y los gobernantes que deben ceñirse a sus textos legislativos y presupuestarios y (4) la presencia de autoridades jurisdiccionales fuertes e independientes» (Duverger, 1995: 138). Problema que incide de forma directa en la organización y la estructura del mercado de trabajo, así como en el diseño de políticas económicas y sociales reductoras de desequilibrios económico-sociales y creadoras de riqueza y bienestar. Se logran así, al menos en teoría, situaciones óptimo paretianas, aunque en la práctica son inalcanzables.

\section{5. ÉTICA, BUEN GOBIERNO Y REPUTACIÓN CORPORATIVA EN LA EMPRESA EUROPEA}

Ho oy en día las corporaciones europeas crean valor que, por definición, son de tres tipos: valor económico, que viene definido según sea el EBITDA que crea la empresa; valor ético, que con un contenido eminente axiológico determina la reputación corporativa y valor profesional, que viene definido según el desempeño profesional y la capacidad de resolución de problemas del capital intelectual que trabaja en la empresa. La obtención de una buena reputación corporativa fideliza a los «stakeholders» de la misma, además de hacer posible nuevas formas de negocio y abrir y/o ampliar nuevos nichos de mercado. El éxito en los negocios viene dado no sólo por la visión personal de un emprendedor, sino también por el equipo humano que está trabajando en la misma línea de negocio. Un equipo humano cohesionado lleva a la creación de una buena reputación corporativa que está arraigada, además, en la cultura corporativa de la empresa.

Dada la fuerte competencia empresarial en el que están inmersas las corporaciones lleva a que la identidad corporativa, definida de forma única y distinguible de la empresa (Gray y Balmer, 1998), constituya un medio para identificar a la empresa de sus competidores.
Identidad corporativa que viene dividida en cultura corporativa y filosofía corporativa. La cultura y filosofía corporativas integran el «know-how», "know-when», "know-what», "know-whom»y «know-who», los cinco, propios de la cultura corporativa, con el «know-why» que caracteriza a la filosofía. Una empresa sin filosofía corporativa camina a la deriva en un mundo empresarial altamente competitivo. Tener claro el porqué se trabaja y hacia dónde se dirige una corporación permitirá a la misma saber cómo contentar a los «stakeholders» de la misma. Por ello, es fundamental preservar dichos valores corporativos mediante la selección de un equipo humano que se sienta identificado con la empresa, ya que los efectos culturales permanecen a largo plazo y son necesarios para lograr que esta alcance unos altos niveles de desarrollo (Cronqvist, Low y Nilsson, 2007).

Las diferencias culturales entre las empresas también vienen determinadas por la elección óptima de incentivos por parte de las corporaciones, así como por el grado de cooperación entre la fuerza de trabajo que labora en la misma. Al ser endógena la utilidad de la cooperación entre el capital humano que trabaja en una 
empresa (Rob y Zemsky, 2000), la corporación es la principal beneficiada, así como sus accionistas a través de la cotización de las acciones de la empresa y la expansión económica de la misma.

El factor cultural también influye en la fidelidad de los empleados a la misma. Mientras que en las empresas europeas es normal y deseable una cierta movilidad laboral, tanto en sentido horizontal como vertical (que oscila, en media, entre cinco y diez años) en las empresas asiáticas la movilidad es mucho menor, e incluso es mal visto por parte de las corporaciones más tradicionales. Esta situación es especialmente palpable tanto en el «keiretsu» japonés (Toyota, Nissan, Sanyo, Sony, Mitsubishi, Mitsui, Sumitomo, Hitachi, Sanwa, Dai-Ichi Kangyo, Fuyo,...$)^{13}$, como en el «chaebol» coreano (Hyundai, Daewoo, Samsung, LG, SK, Hanjin, Lotte, Doosan, Hanhwa, Kumho,...).

En la fidelización influye que toda acción «éticamente orientada puede ajustarse a dos máximas fundamentalmente distintas entre sí e irremediablemente opuestas: puede orientarse conforme a la 'ética de la convicción' ("gesinnungsethisch») o conforme a la 'ética de la responsabilidad' («verantwortungsethisch») (Weber, 1993: 163). Para que se puedan dar ambas de manera simultánea se ha de lograr un elevado grado de fidelización por parte de los empleados y profesionales externos que trabajan en la misma, ya que sólo así se estará a dispuesto a dar lo mejor de ellos mismos para llevar hacia adelante el proyecto empresarial. Sin fidelización ni compromiso por parte de los recursos humanos de la empresa hacia ella misma, es imposible que se pueda dar una ética de la convicción, a pesar de los intentos del Consejo de Administración para lograrlo. En este caso, únicamente quedaría la ética de la respon- sabilidad, sin que ésta sea suficiente para lograr una supervivencia de la corporación a largo plazo.

En cualquier caso y, sobre todo, la ética es personal. Un individuo virtuoso puede sobrevivir en un entorno corrupto, pero una persona corrupta puede infectar el más limpio de los entornos. Por tanto, no llegaremos al núcleo del problema si no nos referimos a los valores que guían a las personas en su toma de decisiones, las bases para sus motivaciones, su personalidad y el carácter que desarrollan como resultado de sus acciones (Fontrodona, 2002). Valores que vienen tomados en función de aspectos antropocéntricos (búsqueda del bienestar del otro), grupales (bienestar del grupo social), familiares (optimización del bienestar familiar) y espirituales (bienestar terrenal y futuro según las creencias religiosas del individuo).

En este sentido y, en lo que respecta a la UE, la influencia del Cristianismo ha sido constante a lo largo de la historia, incluso en las ideas fundacionales de la actual UE (Saiz, 2007 y 2003), a pesar de las corrientes laicistas que han intentado eliminarlo. Así, «la renovación de la visión del mundo por el Cristianismo hace que el modo de pensamiento europeo se desinterese de lo caduco buscando siempre lo mejor, impulsando lo que se llama unas veces la civilización y otras el progreso. Ser libre consiste en tener capacidad de iniciativa para tomar como propias las posibilidades que ofrece una realidad distinta a la del actor y, en principio, 'distante, externa, extraña y ajena' (López Quintás, 2000: 143). Este carácter pragmático de la cultura europea, sustentado en la creencia en que esta vida tiene un valor y realidad propios en orden a la vida futura, se manifiesta en muchos aspectos» (Negro, 2004: 265).

\footnotetext{
${ }^{13}$ El modelo keiretsu japonés se caracteriza, entre los factores más destacables, por estar centralizado en una gran entidad financiera, por existir un férreo control centralizado, y por efectuarse la toma de decisiones en un sentido de arriba hacia abajo. Este modelo tiene su réplica en el Reino Unido (Virgin Group) y la India (Tata Group). También se consideran keiretsu las alianzas aéreas, tales como Oneworld y Star Alliance.
} 
Así mismo y, desde una visión de moral cristiana, «quienes se dedican a la empresa, naturalmente han de obtener ganancias como justa retribución a sus esfuerzos y del servicio que prestan a la sociedad. Pero han de evitar la tentación de buscar el dinero, el poder o el éxito personal por encima de todo» (Echevarría, 2002: 13). Los valores cristianos se traslucen de forma clara en la economía social y en la responsabilidad social de las empresas, ya que en estos casos, el bienestar social impera sobre el meramente económico. Bienestar social en el que los aspectos éticos, basados en una mezcla de religiosidad y humanismo, llevan a que se combinen y se apliquen al unísono, aspectos centrados en Cristo (cristocentrismo) y en el ser humano (antropocentrismo).

A todo sistema ético corresponde un ordenamiento moral (Fernández-Galiano, 1984) basado en la justicia redistributiva y en el buen obrar con una minimización en las pérdidas por mermas o productos defectuosos. Así y, en lo que respecta a la justicia redistributiva, es fundamental «recompensar debidamente a aquellos que han invertido en la empresa su dinero y en ocasiones su tiempo, energía y creatividad» (DelArco, 2006: 135). Sólo así puede cobrar sentido términos tales como solidaridad, cooperación, disminución de brecha norte-sur y justicia social, entre otros.

La globalización económica está acelerando los cambios económicos y sociales en una doble perspectiva Norte-Sur y Este-Oeste. La caída del muro de Berlín en 1989 y del socialismo real ${ }^{14}$ en Europa, por un lado, y la generalización de las TIC, por otro, han acelerado dicho proceso. En realidad, «el proceso de cambio del mercado de trabajo es un continuum. En él confluyen factores económicos, sociales y tecnológicos que, junto con la globalización económica, han acelerado la dinamicidad del proceso y han forzado al mercado para lograr una creciente flexibilidad laboral, tanto horizontal como vertical. Flexibilidad conseguida, en parte, con el auxilio de nuevas tecnologías y la mayor rapidez del transporte» (Saiz, 2004a).

La llegada de las TIC, unido al capital intelectual de las empresas, ha incrementado la importancia de los activos intangibles (entre los que destacan la marca, la reputación corporativa, el «knowhow», la experiencia profesional y las TIC). Los recursos intangibles son estratégicos y contribuyen a alcanzar y mantener ventajas competitivas relacionadas con la rentabilidad empresarial; estas facilitan el crecimiento empresarial orgánico y son estratégicos, al ser difíciles de imitar y copiar de forma aislada; en cuanto a su resultado suele ir unido al del resto de la empresa (Recio, 2005). En consecuencia, la presencia de activos intangibles en la empresa permite a la misma mantenerse en posiciones de liderazgo dentro del sector o industria en donde esté desarrollando su actividad, así como una mayor fidelización de los recursos humanos que trabajan en ella.

Por otra parte, los activos intangibles facilitan a la corporación la realización de prácticas de buen gobierno que mejoran la reputación corporativa de la empresa. Las empresas, con una reputación sólida, son capaces de atraer el mejor talento; sus productos y servicios son percibidos como más valiosos; sus clientes son más fieles $y$ tienen mejores resultados financieros (Eccles, Newquist y Schatz, 2007). Estas ventajas incrementan las posibilidades para que las corporaciones logren niveles de crecimiento sostenible en el tiempo y así beneficien a la sociedad en su conjunto.

\footnotetext{
14 Este fue el término, siguiendo fielmente la doctrina marxista-leninista, utilizado en los países del CAEM (Consejo de Ayuda Económica Mutua) dependientes de la Unión Soviética (1917-1991) durante la Guerra Fría (1945-1991). En Occidente, se utilizó el término «comunismo».
} 
A medida que los activos intangibles van incrementando sus aspectos éticos, mejorará la reputación corporativa, lo que a su vez, mejorará las perspectivas empresariales de crecimiento y de creación de riqueza. Además, al ser la «co-transformadora de la sociedad y, por lo tanto, también co-responsable de llevar a buen término esa transformación» (Del Arco, 2006: 149) tiene un doble fin social y económico, lo que incide en los niveles globales de bienestar social y de creación de riqueza económica. Todo ello llevará hacia la creación de un círculo virtuoso (figura 1) que fortalecerá aún más a los activos intangibles en su conjunto.

Figura 1

El círculo virtuoso de los activos intangibles

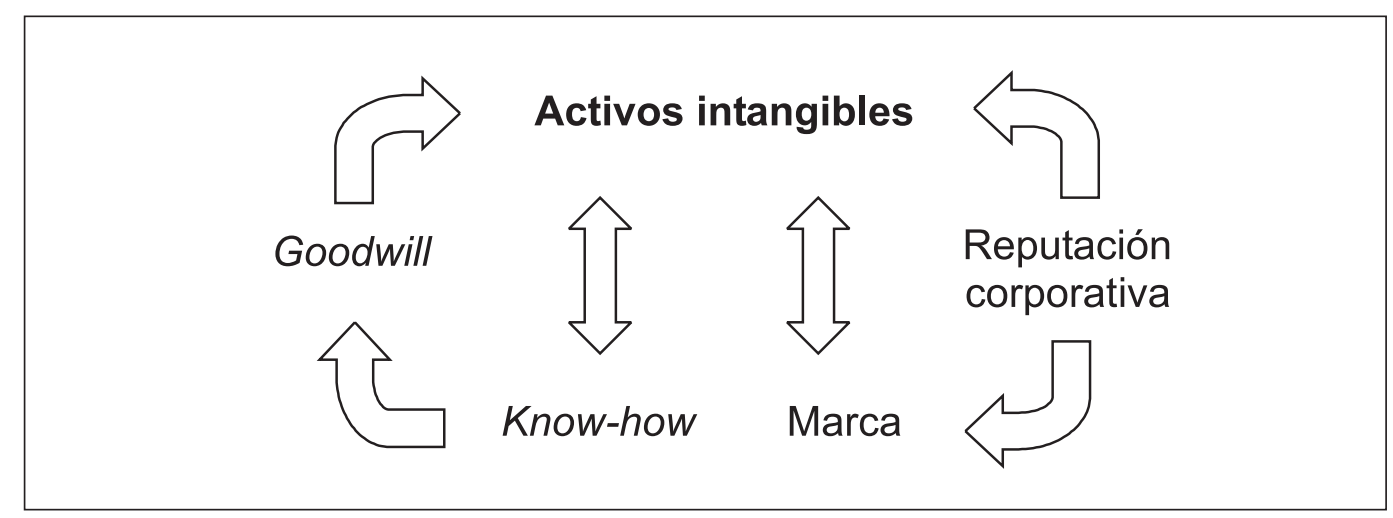

Fuente: elaboración propia

Por otra parte, los activos intangibles inciden directamente en las competencias de la organización, que vienen definidas como «el nivel y los patrones de despliegue de recursos y habilidades pasados y presentes que ayudan a la organización a alcanzar sus metas y objetivos» (Hofer y Schendel, 1978: 25-26).

Activos intangibles que aumentan de valor cuando se producen prácticas de buen gobierno por parte de la empresa. Entre dicho tipo de prácticas se destacan las siguientes:

- La puesta en marcha de políticas de transparencia en la gestión. Se realiza mediante la puesta en público del Balance, Cuenta de Resultados y Cuenta de Pérdidas y Ganancias.

- La definición de objetivos corporativos claros y definidos.
- La realización de una carrera profesional basada en los méritos, la experiencia y el conocimiento.

- Lapreparación de un gabinete de comu-nicación que garantice lafluidezen la recepción de quejas, peticiones y comentarios entre los clientes y la empresa.

- La transparencia en los sueldos por parte del Consejo de Administración, así como la comunicación pública tanto del número de acciones poseídas por el mismo, como de la firma (en su caso) de contratos blindados.

- La obligatoriedad de superar un proceso de auditoria externa con carácter anual.

- La puesta en marcha de políticas de protección a los trabajadores, así como la realización de esfuerzos por parte de la corporación para que sus empleados y profesionales puedan conciliar su vida laboral con la familiar. 
- El establecimiento de uno o varios canales de comunicación, de forma óptima en tiempo real, con los «stakeholders» de la empresa. Una comunicación rápida y fluida mejora la imagen corporativa y aumenta el grado de satisfacción de los «stakeholders» con la empresa.

- El cumplimiento exhaustivo de las obligaciones fiscales con las instituciones y organismos correspondientes.

La realización de prácticas del buen gobierno en las empresas europeas mejora los activos intangibles de estas; aumenta su capitalización bursátil (en caso de cotizar en los mercados financieros); fideliza a los empleados y profesionales que trabajan en la misma; posibilita el logro de sendas de crecimiento sostenible en el tiempo y permite a la empresa alcanzar posiciones de liderazgo en su sector. Las prácticas de buen gobierno, como núcleo de la responsabilidad corporativa, hacen que la empresa consiga unos adecuados niveles de eficacia, seguridad, independencia, transparenciay representatividad que beneficia no sólo a la corporación, sino a la economía y a la sociedad en su conjunto. Permiten, además, a los directivos actuar con racionalidad en entornos competitivos.

En efecto, «cuando el ser humano actúa de manera racional para alcanzar un determinado fin, tiene que asumir que no todos los fines justifican cualquier clase de medios. Hay cierta libertad de elección en la determinación de los fines y los medios, y aquí entra de lleno la ética» (Del Arco, 2006: 142). Como resultado, la ética corporativa tiene una importancia fundamental en todo el proceso de competencia al introducir parámetros de sano comportamiento basados en reglas por cumplir, sin que primen en exclusiva los parámetros darwinianos.

\section{BibliografíA}

CRONQVIST, H.; LOW, A. y NILSSON, M. (2007): «Does Corporate Culture Matter for Firm Policies?», SIFR Research Report Series, 48, Estocolmo (Suecia): Swedish Institute for Financial Research.

DELARCO, J. (2004): Ética para la Sociedad Red, Madrid: Fundación Vodafone \& Dykinson. DUVERGER, M. (1995): Europa de los hombres. Una metamorfosis inacabada, Madrid: Alianza Editorial.

ECCLES, R.G.; NEWQUIST, S.C. y SCHATZ, R. (2007): «Reputation and its Risks 2007», Harvard Business Review, vol. 85 (2), Febrero, pp. 104-114.

ECHEVARRÍA, J. (2002): «Dirigir empresas con sentido cristiano», Revista de Antiguos Alumnos del IESE, 87, septiembre, p. 12-15.

FERNÁNDEZ-GALIANO, A. (1986): Derecho Natural. Introducción filosófica al Derecho, quinta edición, Madrid: CEURA 
FOMBRUN, C.J. y SHANLEY, M. (1990): «What's in a Name: Reputation-building and Corporate Strategy», Academy of Management Journal, 33, pp. 233-258.

FONTRODONA, J. (2002): «Lecciones éticas del caso Enron», Revista de Antiguos Alumnos del IESE, 87, septiembre, p. 20.

GARCÍA MENÉNDEZ, J.R. (1996): «Neoproteccionismo, dumping social y eco-dumping», Nueva Sociedad, núm. 143, Junio, pp. 124-141.

GRAY, E. y BALMER, J. (1998): «Managing Corporate Image a Corporate Reputation», Long Range Planning, vol. 31, núm. 5, pp. 695-702.

HOFER, C.W. y SCHENDEL, D.E. (1978): Strategy Formulation: Analytical Concepts, West Publishing, St. Paul.

Institut der deutschen Wirtschaft (IDW) Köln (2007): „Zu viel Korruption und zu wenig Bildung», núm. 23, Colonia (Alemania), pp. 4-5.

MATHIS, J.H. (2003): «Principios Fundamentales y prohibición en el marco de la OMC: obligaciones relativas a las prácticas privadas, leyes nacionales sobre la competencia y consecuencias para un marco de política de competencia», Documento interno, Conferencia de las Naciones Unidas sobre Comercio y Desarrollo, Ginebra (Suiza).

NAVAS, J.E. y GUERRAS, L.A. (1998): La Dirección Estratégica de la Empresa, Madrid: Civitas. NEGRO, D. (2004): Lo que Europa debe al Cristianismo, Madrid: Unión Editorial.

QUEVEDO, E. de (2003): Reputación y creación de valor: una relación circular, Madrid: Thomson. RECIO, M.L. (2005): Los recursos intangibles: gestión y reconocimiento en la empresa española, Madrid: Instituto de Estudios Económicos.

SAIZ, J.M. (2007): «La visión cristiana de los padres de Europa», UNISCI Discussion Papers, 14, Mayo, Facultad de Ciencias Políticas y Sociología, Universidad Complutense de Madrid, pp. 115-129.

— (2006): «Ética empresarial y lucha contra el fraude en la UE», en Galindo, M.A. (Dir): Ética y economía, Colección Estudios de Hacienda Pública, Madrid: Instituto de Estudios Fiscales, pp. 249-270.

- (2004a): «Nuevas Tecnologías y Mercado de Trabajo. Situación actual de la externalización de servicios», I Congreso Internacional sobre Tecnología Documental y del Conocimiento, Madrid.

- (2004b): Claves para un nuevo mercado de trabajo. Una aplicación a la Unión Europea, Alicante (España): Editorial Club Universitario.

- (2003): «Reflexiones sobre la unificación europea desde una perspectiva cristiana», en $V$ Congreso "Católicos y Vida Pública», Universidad San Pablo-CEU, Madrid, 14-16 de noviembre. WEBER, M. (1993): El político y el científico, Madrid: Alianza(Footnotes) 


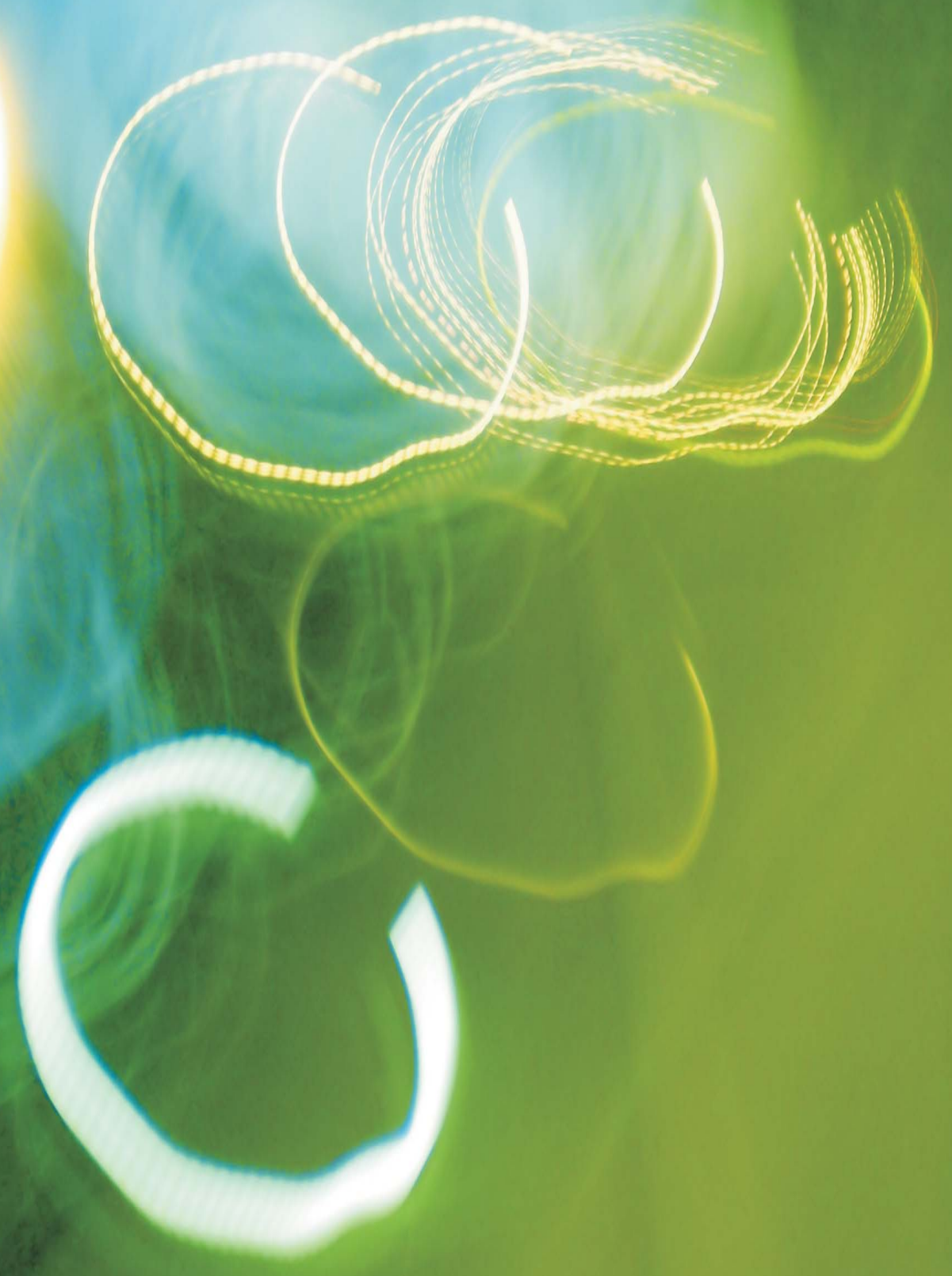

